Planetary Systems in the Universe - Observation, Formation and Evolution

Proceedings IAU Symposium No. 202, (C)2004 IAU

Alan Penny, Pawel Artymowicz, Anne-Marie Lagrange, $\mathcal{G}$ Sara Russell, eds.

\title{
Giant planet formation - a theoretical timeline
}

\author{
Günther Wuchterl \\ Max-Planck-Institut für extraterrestrische Physik, Giessenbachstraße, \\ D-85748 Garching, Germany, http://youngstars.mpe.mpg.de/
}

\begin{abstract}
Low mass circumstellar disks are a result of the star formation process. The growth of dust and solid planets in such pre-planetary disks determines many properties of our solar system. Models of the Solar System giant planets indicate an enrichment of heavy elements and imply heavy element cores. Detailed models therefore describe giant planet formation as a consequence of the formation of solid planets that have grown sufficiently large to permanently bind gas from the protoplanetary nebula. The diversity of Solar System and extrasolar giant planets is explained by variations in the core growth rates caused by a coupling of the dynamics of planetesimals and the contraction of the massive envelopes they dive into, as well as by changes in the hydrodynamical accretion behavior of the envelopes resulting from differences in nebula density, temperature and orbital distance. Detailed formation models are able to determine observables as luminosities, radii and effective temperatures of young giant planets. Present observational techniques do now allow to probe star formation regions at ages covering all evolutionary stages of the giant planet formation process.
\end{abstract}

\section{Introduction}

The understanding of giant planet formation is based on the observational study of planets in the solar system and in orbit around other stars as well as the application of physical principles to cosmogony. Observational constraints can be obtained from studies of solar system planets that provide information about their composition and radial structure at ages of 4.6 Gyr (see Guillot's this volume). The analyses of meteorites provide information about the ages and formation- and life-time ranges for the various nebula-components, in particular solids, mostly in the region of the asteroid belt (Russel and Robert, this volume), that may be taken to extrapolate for the respective properties in the giant planet formation region. Constraints from the observations of disks around young stars (Nakagawa and Beckwith, this volume) are relatively weak due to resolution limits of present telescopes (Dutrey 1999) and essentially give estimates for the disk/star mass ratio in the range that was theoretically expected and lifetime-estimates for components of the nebulae (Beckwith, this volume). Further information about conditions in and evolution of the protoplanetary disks can also be sought from a comparison of model chemical histories to the isotopic ratios measured now in the solar system (e.g. Drouart et al. 1999). Basic physical principles and the theoretical modeling of key uncertain processes, 


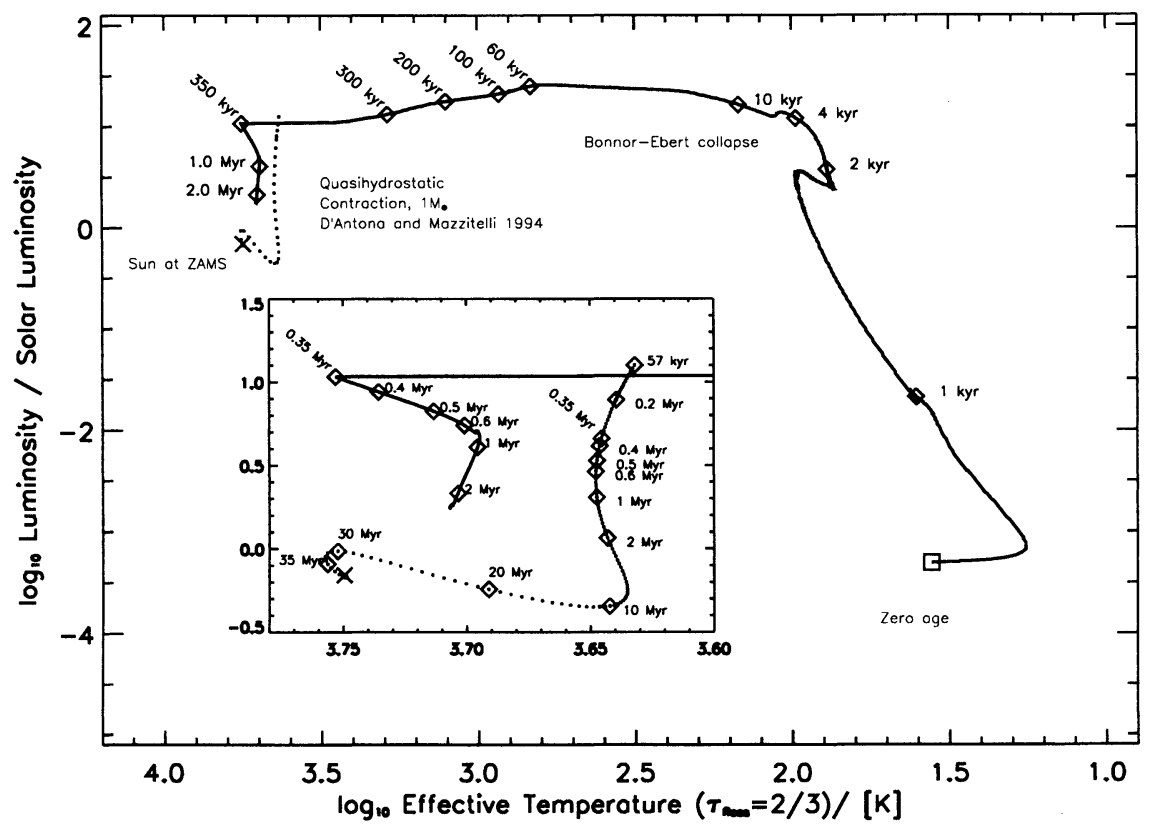

Figure 1. Theoretical Hertzprung-Russel diagram for the collapse of a Bonner-Ebert-sphere with an initial temperature of $10 \mathrm{~K}$ and a solar mass. Ages that are measured from the moment of formation of the first photosphere are labeled in years along a radiation-fluid-dynamical evolutionary track (full line). A hydrostatic stellar-evolution calculation of a solar mass (D'Antona and Mazzitelli 1994, MLT,Alexander-case) is shown for comparison (dotted).

such as the anomalous ${ }^{1}$ nebula viscosity required to solve the stellar angular momentum problem and allow accretion through a disk, provide information about the collapse of the parent interstellar cloud fragments and the structure of the pre- and protoplanetary nebulae. The theory of star formation and early stellar evolution provides evolutionary time-scales for the star-nebula systems and a clock for empirical studies of giant planet formation and protoplanetary disk evolution. Via the properties of the young stars and star-disk interactions, nebula conditions and nebula evolution depend on early stellar evolution. Radiation fluid dynamical calculations address the question of whether giant planets might form without the gravity-enhancement of large solids by a triggered dynamical instability of the nebula disk (Boss, this volume).

Planetesimal dynamics in the nebula determines accretion-rates of solid bodies larger than a few kilometers, that grow by mutual collisions and the time-scales required for their growth to planetary masses (Ida, this volume). Calculations of the structure of the envelope equilibria of protoplanets embedded

\footnotetext{
${ }^{1}$ i.e. turbulent due to fluid-dynamical or magneto-hydrodynamical instabilities or effective due to large scale flow structure such as spiral shocks or gravitational torques
} 
in the nebula determine the solid masses required to permanently bind gases of the otherwise gravitationally stable protoplanetary disk to 'cores'. Radiation fluid dynamics of proto giant planet envelopes and -environments determines if, when and how sufficiently large proto giant planets can grow to mature giant planets with masses comparable to Jupiter (Wuchterl, Guillot \& Lissauer 2000). It also determines the thermal input, that starts the subsequent evolution of the giant planet by contraction and cooling at constant mass, and the thermal excessemission that still can be measured for solar system giant planets (Burrows, this volume).

I will mostly put some results in giant planet formation in a theoretical framework that provides the context to build up a coherent chronology from the earliest cloud states to main sequence stars and I will point out the properties of young stars at the time when planet formation theories expect the earliest planets.

\section{A time-line for solar system formation}

The general problem of the evolution from cloud collapse to a mature star on the main sequence, that replenishes all energy radiated into space from the surface, by nuclear reactions in the interior, is still unsolved and usually the evolution is split into various parts that are discussed using separate and often timeindependent (steady or static) models (e.g. Palla and Stahler 1991, 1992). Hence there is an understanding of the important processes (e.g. Hayashi et al. 1985) but no coherent dynamics from the protostellar collapse to the main-sequence and therefore no coherent time-evolution and as a consequence no 'clock' for solar system formation.

Recently Wuchterl \& Tscharnuter (2001), see also Wuchterl (2000a) have given a set of equations that, admittedly simple due to the assumed spherical symmetry, contains both, the essential stellar physics (radiation, gravity, convection, nuclear reactions) and the basics of cloud physics and collapse (radiation fluid-dynamics, low optical depth radiative transfer, supercritical shocks). These calculations determine the evolution of a spherical isothermal cloud (a BonnorEbert sphere) from gravitational instability to stellar evolution phases beyond deuterium burning. The time-evolution of such a calculation is shown in Fig. 1. One might think that a Bonner-Ebert sphere is a very idealized cloud, but recent observations show clear evidence for the de facto existence under realistic conditions (Alves et al. 2001). Respective calculations of the collapse of a turbulent fragmenting cloud resulting in the formation of a cluster lead to a similar picture (Wuchterl \& Klessen 2001) and essentially the same stellar properties at an age of 1 Myr.

Stellar zero age is put here to the moment when the cloud becomes optically thick at the frequences that govern energy transfer in the densest part, i.e. when the Rosseland mean optical dept of the cloud center reaches $2 / 3$ (Wuchterl \& Tscharnuter 2001). After that moment an optically thin photosphere can be distinguished from an optically thick inner part, the stellar embryo. Once the cloud center becomes optically thick energy cannot escape from the cloud directly as fast as it is produced - as in the isothermal phase of the cloud collapse - but is retained and hence a thermal reservoir forms. The content 


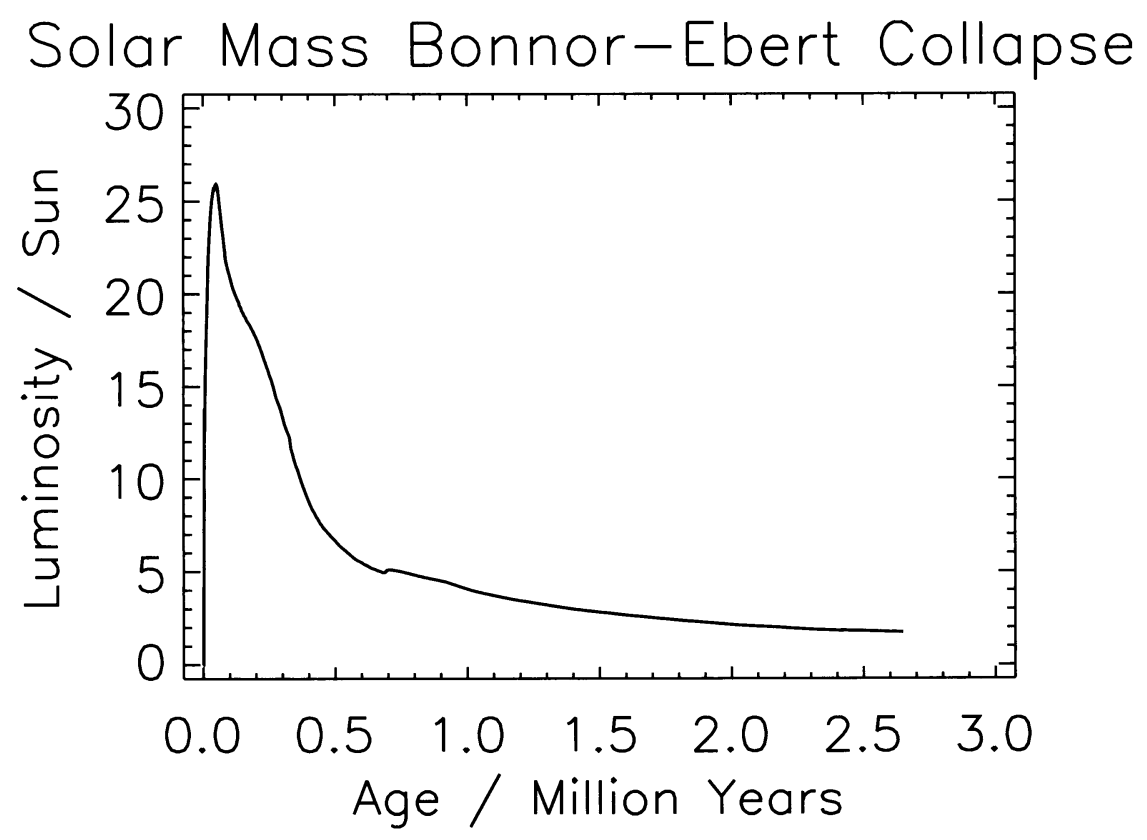

Figure 2. Luminosity of a solar mass protostar/young star during the aera of planet formation. The evolution starts at zero luminosity with an isothermal cloud fragment (marginally gravitationally unstable Bonner Ebert sphere at $10 \mathrm{~K}$ ) and ends with a solar mass young star in the pre-main sequence phase.

of this thermal reservoir depends on the initial input and the surface losses and can be used as a clock. This is the only astrophysical clock that is available in young star forming regions that is practically accessible by observations of stellar properties and that covers the entire time-span to the main-sequence. The Kelvin-Helmholtz contraction during the pre-main sequence phase essentially is the time-evolution of young stars after they have been formed.

\subsection{Formation of the preplanetary nebula and rocks}

To link this astrophysical clock to the meteoritic clock based on radioactive dating, an important question is now at what stage the rocks or grains will be formed. If we are interested in 'nebula products' a necessary condition for that is the formation of a preplanetary nebula. The question of when this happens cannot be addressed by a calculation in spherical symmetry, but we can look for the evolutionary status of the central protostar in the spherical calculation and compare it to the corresponding state in e.g. an axisymmetric collapsecalculation, that shows the formation of the preplanetary nebula (Tscharnuter 1987). The comparison shows that the nebula forms within a few thousand years after the second collapse of the core comes to a halt and the final hydrostatic protostellar core settles into hydrostatic equilibrium and the quasi-steady main accretion phase starts. This puts the moment of the formation of the preplan- 

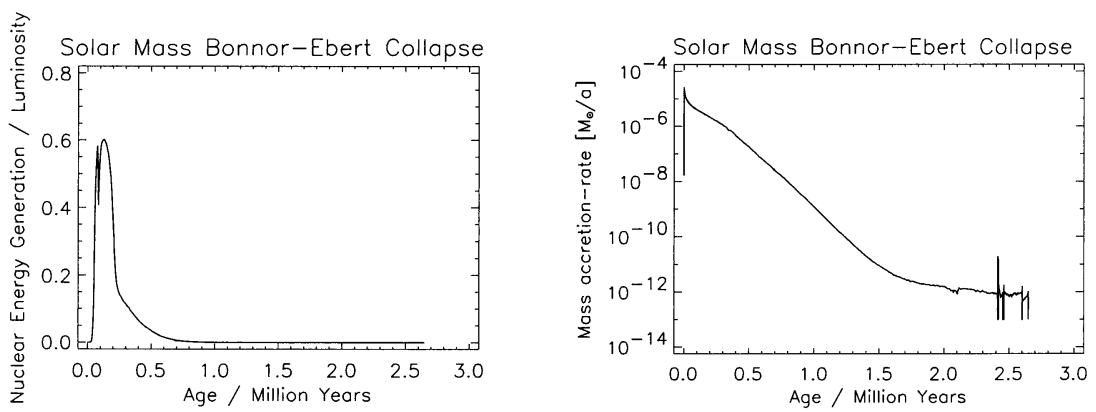

Figure 3. Formation and early evolution of a solar mass star at ages relevant for planet formation as calculated from the collapse of a Bonnor-Ebert sphere. (a) Fraction of the stellar/protostellar luminosity due to Deuterium burning as a function of age. (b) Mass accretion-rate at the stellar photosphere (Rosseland mean optical depth equal $2 / 3$ ), in units of solar masses per year, as a function of age.

etary nebula to slightly earlier than at the $10 \mathrm{kyr}$ mark on the track in Fig. 1. That is $10 \mathrm{kyr}$ after the formation of the first photosphere. Within the dense flattened nebula-disk solids can grow to rock sizes on a kyr time-scale, and hence I would suggest to assign that age-mark to the oldest nebula products. Due to the simplifications of spherical collapse that is most likely a lower limit since spherical accretion has probably the highest efficiency and hence leads to the fastest evolution, and the most rapid clock. This earliest phase in the evolution of a disk is also the moment when gravitational instabilities of more massive disks are most likely to operate, if they indeed occur (see Boss, this volume).

\subsection{Planetesimal formation and runaway growth}

The maximum luminosity of the protostar is reached at $60 \mathrm{kyr}$ (cf. Fig. 2), hence there is a significant period while nebula temperatures are likely to increase with time at a given position. At $100 \mathrm{kyr}$ - a typical time for a planetesimal at terrestrial planet orbital radii to grow to a planetary embryo at the end of runaway growth (see e.g. Ida, this volume, Lissauer 1993) — essentially all deuterium has been burnt in the stellar embryo (cf. Fig. 3) and the luminosity starts to decrease as a consequence of the now significant depletion of the mass in the free-falling envelope as a large fraction of the original cloud fragment ist already in the central protostar. The optical depth of the cocoon decreases and hence photons come from deeper and hotter regions of the protostellar envelope, and the effective temperature rises despite the decrease in luminosity due to the fading of accretion (cf. Figs. 1 and $3 \mathrm{~b}$ ). At $300 \mathrm{kyr}$ the central protostar is still hidden in the cocoon. As the latter is further diluted it becomes transparent and photons can escape directly from the stellar photosphere and the star becomes visible at the corner, of maximum temperature at $350 \mathrm{kyr}$ in Fig. 1. Hence it is plausible that the runaway phases are likely to be completed in large parts of the nebula, when the central star becomes visible, especially when considering the fast clock spherical accretion provides. It may be noted that the luminosity is still ten times solar at this point. Mass accretion-rates have dropped considerably 


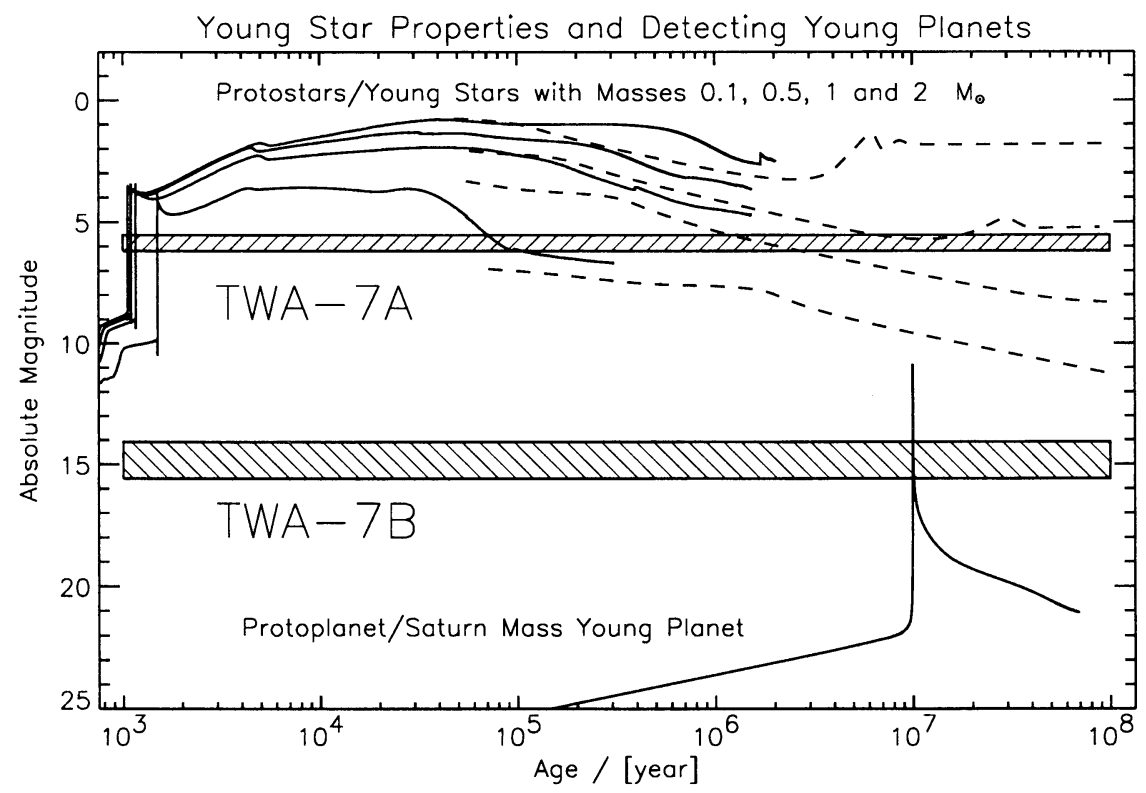

Figure 4. Absolute magnitudes of the nearby young star TWA-7A and candidate companion TWA-7B (that was rejected as a background object after spectroscopy by Neuhäuser et al. 2000) compared to protostars and young stars of various masses (models from Wuchterl \& Tscharnuter 2001, full lines and D'Antona \& Mazzitelli, dashed lines) and a saturn-mass protoplanet (Wuchterl 2000b)

due to the approach of the start to its final mass and the respective depletion of the envelope mass (see Fig. 3b). At 550 Myr the star has accreted $99 \%$ of it final mass of $1 \mathrm{M}_{\odot}$, but the remaining percent is still comparable to the minimum mass nebula and upon inspection of the mass accretion-rate it is clear that there is time to grow a few generations of runaway embryos by resupplying the nebula from the envelope in the late accretion phases of the star, or rather to prolong the runaway phase, that usually ends when the feeding zone is emptied, by the generation of new planetesimals.

\subsection{Protoplanetary envelope capture and the critical mass}

The end of the runaway phase of planetesimal accretion is of key importance in the formation of giant planets (cf. Ida, this volume, Lissauer 1993, Wuchterl et al. 2000). At that evolutionary phase planetary formation changes its speed relative to the evolution of the central star. In the earlier phases, that last a few $10^{5}$ years, planetesimals and planetary embryos grow faster than the star is accreting and contracting (cf. Figs 2, 3b). When a growing protoplanetary embryo has no planetesimals remaining in its accretion-range, embryo growth becomes much slower. After that moment planetary embryos have to perturb 
each other into crossing orbits by mutual gravitational perturbations (cf. Ida's contribution). A key question is therefore whether the mass of the planetary embryos at this moment is smaller or larger than the so-called critical or crossover mass necessary to efficiently accrete nebula gas and hence for the growth to giant planet masses. Typically the critical mass is a few earth masses (cf. Wuchterl et al. 2000) and larger than the isolation mass and giant planet formation is thought to involve a phase of slow growth (cf. Ida's contribution). Therefore it is often argued that there is a time-scale problem for giant planet formation (e.g. Boss's contribution). But the time needed to reach the critical mass and to form a giant planet is reduced if (1) surface densities are enhanced relative to the values of the minimum reconstitutive mass solar nebula (see e.g. Lissauer 1993), (2) the isolation of the embryo is impeded, e.g. by orbital migration (see Ida's contribution), or (3) the critical mass is reduced under certain nebula conditions (e.g. Wuchterl 1993, Wuchterl et al. 2000). If we compare the timescales of star-formation and early stellar evolution, as discussed above, another possibility is the addition of new material to the nebula while planet formation is in progress. That seems likely to occur according to the timeline given above for the formation of a solar mass star in spherical symmetry, that is probably faster than disc-accretion that is slowed down by the relatively inefficient angular momentum transfer processes that control disc-evolution.

\subsection{Accretion of giant planet envelopes}

After passing the bottleneck of assembling a sufficiently large core to start efficient gas-accretion, giant planet formation becomes a relatively rapid process that occurs on the thermal contraction time-scale (Bodenheimer \& Pollack 1986) of about a Myr or the much shorter dynamical time-scale of the gaseous envelope (Wuchterl 1991, 1995b, Boss, this volume). If the growth of the planet is controlled by gas accretion through a gap formed by the interaction of the protoplanet with the nebula disk, giant planet growth-times depend on the nebula viscosity and are of the order of $10^{4}-10^{5}$ years (e.g. Kley 1999, Artymowicz's contribution).

\section{Direct detection of planets of nearby young stars}

Neuhäuser et al. (2000, and this volume) have recently shown that the direct detection of young extrasolar planets is possible with existing telescopes from the ground. In Fig. 4 the various evolutionary phases of giant planet evolution are shown for the case of an ultimately saturn-mass protoplanet. To correctly represent the rise and the fall of the planet's luminosity as a function of age (cf. Fig. 5) it is necessary to consider the formation process. That in turn is important for estimating planetary masses from luminosities at very young ages that are now accessible by observations.

\section{Conclusion}

Giant planet formation can now be viewed in the theoretical evolutionary framework of dynamical star-formation theory and the key phases of giant planet 
formation that decide about overall formation-time are presently becoming accessible to observation. An evolutionary picture of concurrent star and planet formation is presently at an early stage and hence it is probably premature to decide about a time-scale problem for giant planet formation in that framework. But both observational and theoretical progress require the study of planet formation together with an evolving star and an evolving nebula. Giant planet formation is at the verge of becoming directly accessible to observational study that will provide a test for the theories developed up to now mostly for the formation of the solar system.

\section{References}

Alves, J. F., Lada, C. J., \& Lada, E. A. 2001, Nature, 409, 159

Bodenheimer, P., \& Pollack, J. B. 1986, Icarus, 67, 391

D'Antona, F. \& Mazzitelli, I. 1994, ApJS, 90, 457

Dutrey, A. 1999, in Planets Outside the Solar System: Theory and Observations, ed. J.-M. Mariotti \& D. Alloin. (Dordrecht: Kluwer), 13

Drouart, A., Dubrulle, B., Gautier, D., \& Robert, F. 1999, Icarus, 140, 129

Kley, W. 1999, MNRAS, 303, 696

Lissauer, J. J. 1993, ARA\&A, 31, 129

Neuhäuser, R., Brandner, Eckart, Guenther, Alves, Ott, Huelamo, \& Fernandez 2000, A\&A, 354, L9

Palla, F., \& Stahler, S. W. 1991, ApJ, 375, 288

Palla, F., \& Stahler, S. W. 1992, ApJ, 392, 667

Tscharnuter, W.M. 1987, A\&A, 188, 55

Wuchterl, G. 1991b, Icarus, 91, 53

Wuchterl, G. 1995b, Earth Moon and Planets, 67, 51

Wuchterl, G. 1993, Icarus, 106, 323

Wuchterl, G. 2000a, In Cool Stars, Stellar Systems and the Sun, Challenges for the New Millenium, the XI Cambridge workshop, ed., R.J. Garcia Lopez, R. Rebolo \& M.-R. Zapatero-Osorio, (San Francisco: ASP), in press ${ }^{2}$

Wuchterl, G. 2000b, in From Extrasolar Planets to Cosmology: The VLT Opening Symposium, ed. J. Bergeron \& A. Renzini 408(Berlin: Springer)

Wuchterl, G., Guillot, T., \& Lissauer, J.J. 2000, in Protostars and Planets IV, ed. V. Mannings, A. P. Boss \& S. S. Russell, (Tucson: University of Arizona Press), 1081

Wuchterl, G., \& Klessen R. 2001, ApJ, subm. ${ }^{2}$

Wuchterl, G., \& Tscharnuter, W.M. 2001, A\&A, subm. ${ }^{2}$

\footnotetext{
${ }^{2}$ Pre/Reprints are available via http://youngstars.mpe.mpg.de/
} 